\title{
A Probabilistic Counting Lemma for Complete Graphs
}

\author{
Stefanie Gerke, Martin Marciniszyn and Angelika Steger \\ Institute of Theoretical Computer Science, ETH Zürich, CH-8092 Zürich, Switzerland
}

\begin{abstract}
We prove the existence of many complete graphs in almost all sufficiently dense partitions obtained by an application of Szemerédi's Regularity Lemma. More precisely, we consider the number of complete graphs $K_{\ell}$ on $\ell$ vertices in $\ell$-partite graphs where each partition class consists of $n$ vertices and there is an $\varepsilon$-regular graph on $m$ edges between any two partition classes. We show that for all $\beta>0$, at most a $\beta^{m}$-fraction of graphs in this family contain less than the expected number of copies of $K_{\ell}$ provided $\varepsilon$ is sufficiently small and $m \geq C n^{2-1 /(\ell-1)}$ for a constant $C>0$ and $n$ sufficiently large. This result is a counting version of a restricted version of a conjecture by Kohayakawa, Łuczak and Rödl [8] and has several implications for random graphs.
\end{abstract}

\section{Introduction}

The celebrated Regularity Lemma by Szemerédi [15] states, roughly speaking, that the vertex set of every sufficiently large graph can be partitioned into a constant number of classes in such a way that the edges between most partition classes are distributed regularly. It is particularly useful for finding subgraphs in large graphs since one can employ the structure of the partition obtained from the lemma and show the existence of the subgraph in there. Lemmas that assert the existence of subgraphs in such a partition are called embedding or counting lemmas. For numerous applications of Szemerédi's Regularity Lemma and various powerful embedding lemmas for dense graphs, we refer the reader to the excellent survey articles [11, 12].

We are interested in a counting lemma for sparse graphs. It is well known that Szemerédi's original lemma is only helpful for dense graphs (that is graphs with $n$ vertices and $\Theta\left(n^{2}\right)$ edges), but Kohayakawa [7] and Rödl (unpublished) independently introduced a variant of Szemerédi's Regularity Lemma for sparse graphs. Unfortunately, there do not (yet) exist corresponding counting or embedding lemmas, and moreover one can show that straight-forward generalizations of these are false as was noted by Łuczak (see [5, 9]). However, Kohayakawa, Łuczak, and Rödl [8] formulated a probabilistic embedding lemma (see Conjecture 3) that, if true, would solve some long-standing open problems in random graph theory [5, $6,8,13]$.

Conjecture 3, an embedding lemma, states the existence of a fixed graph $H$ in a certain structure. We generalize this conjecture to a counting version and ask for several copies of $H$ in this structure. We then prove this generalized version for some special cases. In order to state the conjecture, we need the following definitions. 
Definition 1 For a graph $G=(V, E)$ and two disjoint sets $V_{1}, V_{2} \subseteq V$, we denote the set of edges with one endpoint in $V_{1}$ and one endpoint in $V_{2}$ by $E\left(V_{1}, V_{2}\right)$. The density $d\left(V_{1}, V_{2}\right)$ is defined as $d\left(V_{1}, V_{2}\right)=$ $\left|E\left(V_{1}, V_{2}\right)\right| /\left(\left|V_{1}\right|\left|V_{2}\right|\right)$. We say that the graph induced by $V_{1}, V_{2}$ is $(\varepsilon)$-regular if for all $V_{1}^{\prime} \subseteq V_{1}$ and $V_{2}^{\prime} \subseteq V_{2}$ with $\left|V_{1}^{\prime}\right| \geq \varepsilon\left|V_{1}\right|$ and $\left|V_{2}^{\prime}\right| \geq \varepsilon\left|V_{2}\right|,\left|d\left(V_{1}^{\prime}, V_{2}^{\prime}\right)-d\left(V_{1}, V_{2}\right)\right| \leq \varepsilon d\left(V_{1}, V_{2}\right)$.

Note that a bipartite graph $B=\left(V_{1} \cup V_{2}, E\right)$ is $(\varepsilon)$-regular if for all sufficiently large sets $V_{1}^{\prime}$ and $V_{2}^{\prime}$ the density is only $\varepsilon d\left(V_{1}, V_{2}\right)$ away from the density of the graph. In particular, the smaller the density of the underlying graph and $\varepsilon$ are, the closer are the densities of large sets of an $(\varepsilon)$-regular graph to their expected value.

We have defined $(\varepsilon)$-regularity for pairs of vertex sets, but we are mainly interested in $\ell$-partite graphs such that certain pairs of partition classes form an $(\varepsilon)$-regular graph.

Definition 2 For a graph $H$, let $\mathcal{G}(H, n, m)$ be the family of graphs on the vertex set $V=\bigcup_{x \in V(H)} V_{x}$ with pairwise disjoint sets of vertices $V_{x}$ of size $n$ and the edge set $E=\bigcup_{\{x, y\} \in E(H)} E_{x y}$, where $E_{x y} \subseteq$ $V_{x} \times V_{y}$ and $\left|E_{x y}\right|=m$. Let $\mathcal{G}(H, n, m, \varepsilon) \subseteq \mathcal{G}(H, n, m)$ denote the set of graphs in $\mathcal{G}(H, n, m)$ satisfying that each $\left(V_{x} \cup V_{y}, E_{x y}\right)$ is an $(\varepsilon)$-regular graph.

Note that a graph $G \in \mathcal{G}(H, n, m, \varepsilon)$ looks like $H$ with every vertex blown up to a class of $n$ vertices and every edge to a set of $m$ edges, which form an $(\varepsilon)$-regular graph between the corresponding vertex classes. One may obtain graphs of $\mathcal{G}(H, n, m, \varepsilon)$ by applying Szemerédi's Regularity Lemma to a large graph $G$ (and removing some edges). Thus, if one wants to find a copy of $H$ in $G$, it suffices to find $H$ in all graphs of $\mathcal{G}(H, n, m, \varepsilon)$. As we have already mentioned, this is not always possible if $m=o\left(n^{2}\right)$. Moreover, if one considers graphs in $G(H, n, m)$, then one can show that there exists a constant $c$ such that at least a $c^{m}$ fraction does not contain $H$. Kohayakawa, Łuczak and Rödl conjectured that the fraction of graphs in $G(H, n, m, \varepsilon)$ that does not contain a graph $H$ is substantially smaller.

\section{Conjecture 3 (KŁR-Conjecture [8]) Let $H$ be a fixed graph and let}

$$
\mathcal{F}(H, n, m)=\{G \in \mathcal{G}(H, n, m): H \text { is not a subgraph of } G\} .
$$

For any $\beta>0$, there exist constants $\varepsilon_{0}>0, C>0, n_{0}>0$ such that for all $m \geq C n^{2-1 / d_{2}(H)}, n \geq n_{0}$, and $0<\varepsilon \leq \varepsilon_{0},|\mathcal{F}(H, n, m) \cap \mathcal{G}(H, n, m, \varepsilon)| \leq \beta^{m}\left(\begin{array}{c}n^{2} \\ m\end{array}\right)$, where

$$
d_{2}(H)=\max \left\{\frac{|E(F)|-1}{|V(F)|-2}: F \subseteq H,|V(F)| \geq 3\right\} .
$$

Conjecture 3 can easily be verified for trees and it is also known to be true when $H$ is a cycle [1] or when $H$ is the complete graph on 3 [13], 4 [3] and 5 [4] vertices.

Our main result is a counting version of Conjecture 3 for $H$ being the complete graph $K_{\ell}$ of size $\ell$ and $m \geq C n^{2-1 /(\ell-1)}$ (instead of $m \geq C n^{2-2 /(\ell+1)}$ as stated in Conjecture 3). In order to state it, we need the following definition.

Definition 4 We denote the family of graphs in $\mathcal{G}(H, n, m)$ that contain less than $(1-\delta) n^{|V(H)|}\left(m / n^{2}\right)^{|E(H)|}$ copies of $H$ by $\mathcal{F}(H, n, m, \delta)$.

Observe that apart from the factor $(1-\delta)$ this quantity is equal to the expected number of subgraphs isomorphic to $H$ in $G \in \mathcal{G}(H, n, m)$ if the edges are randomly distributed between the vertex classes. We conjecture that one can replace $\mathcal{F}(H, n, m)$ by $\mathcal{F}(H, n, m, \delta)$ in Conjecture 3 . 
Conjecture 5 (Counting Lemma) Let $H$ be a fixed graph. For any $\beta>0$ and $\delta>0$, there exist constants $\varepsilon_{0}>0, C>0, n_{0}>0$ such that for all $m \geq C n^{2-1 / d_{2}(H)}, n \geq n_{0}$, and $0<\varepsilon \leq \varepsilon_{0}$,

$$
|\mathcal{F}(H, n, m, \delta) \cap \mathcal{G}(H, n, m, \varepsilon)| \leq \beta^{m}\left(\begin{array}{c}
n^{2} \\
m
\end{array}\right)^{|E(H)|} .
$$

Our main result states that Conjecture 5 (and thus Conjecture 3) holds true if $H$ is a complete graph and $m$ is slightly greater than conjectured.

Theorem 6 For all $\ell \geq 3, \delta>0$, and $\beta>0$, there exist constants $n_{0} \in \mathbb{N}, C>0$, and $\varepsilon>0$ such that

$$
\left|\mathcal{F}\left(K_{\ell}, n, m, \delta\right) \cap \mathcal{G}\left(K_{\ell}, n, m, \varepsilon\right)\right| \leq \beta^{m} \cdot\left(\begin{array}{c}
n^{2} \\
m
\end{array}\right)^{\left(\begin{array}{l}
\ell \\
2
\end{array}\right)}
$$

provided that $m \geq C n^{2-1 /(\ell-1)}, n \geq n_{0}$, and $0<\varepsilon \leq \varepsilon_{0}$.

It is well known $[6,8,13]$ that Conjecture 3 has several implications. In particular Theorem 6 implies the following.

Corollary 7 [13] For all $\ell \geq 3$ and every $\delta>0$, there exists $c=c(\delta, \ell)$ such that the probability that a graph chosen uniformly at random from the family of all $K_{\ell}$-free labeled graphs on $n$ vertices and $m \geq c n^{2-1 /(\ell-1)}$ edges can be made $(\ell-1)$-partite by removing $\delta m$ edges tends to one as $n$ tends to infinity.

Corollary 8 [13] For all $\ell \geq 3$ and for every $\varepsilon>0$, there exist $c=c(\varepsilon, \ell)$ and $n_{0}=n_{0}(\varepsilon, \ell)$ such that for $n \geq n_{0}$ and $c n^{2-1 /(\ell-1)} \leq m \leq n^{2} / c$, a graph $G_{n, m}$ drawn uniformly at random from all labeled graphs on $n$ vertices and $m$ edges satisfies

$$
\left(\frac{\ell-2}{\ell-1}-\varepsilon\right)^{m} \leq \mathbb{P}\left(G(n, m) \text { does not contain } K_{\ell}\right) \leq\left(\frac{\ell-2}{\ell-1}+\varepsilon\right)^{m}
$$

Another implication of Theorem 6 concerns the maximal number of edges $\operatorname{ex}(G, H)$ that a subgraph of $G$ may have without containing a copy of $H$ if $G=G_{n, p}$. As usual, $G_{n, p}$ is the probability space of all graphs on $n$ labeled vertices, where each edge is present with probability $p$ independently of all other edges. Let $\ell \geq 3$ and $0<p=p(n) \leq 1$ be such that $p n^{1 /(\ell-1)} \rightarrow \infty$ as $n \rightarrow \infty$. Then asymptotically almost surely (a.a.s.), that is, with probability tending to one as $n$ tends to infinity

$$
\operatorname{ex}\left(G_{n, p}, K_{\ell}\right) \leq\left(1-\frac{1}{\ell-1}+o(1)\right)\left(\begin{array}{l}
n^{2} \\
m
\end{array}\right)^{\left(\begin{array}{c}
\ell \\
2
\end{array}\right)}
$$

This result was already shown in $[10,14]$. Let us remark that using Theorem 6 together with the sparse version of the Regularity Lemma in fact implies a slightly stronger result. Namely, we obtain that a.a.s. every subgraph of $G_{n, p}$ with $(1+\delta) \operatorname{ex}\left(G_{n, p}, K_{\ell}\right)$ edges contains not just one copy of a $K_{\ell}$ (which is the essential statement of the above result) but $c_{\delta} n^{\ell} p^{\ell(\ell-1) / 2}$ many copies.

We shall prove Theorem 6 by induction on $\ell$. Let us remark that the lower bound on $m$ in the theorem depends on the base case of the induction which is $\ell=3$ in our proof. If we want to prove Conjecture 3 , we can chose $\ell=5$ as the base case for the induction since Conjecture 3 has been verified in this setting 
in [4]. The induction would work analogously and yield the threshold $m \geq n^{2-1 /(\ell-2)}$. This includes the main result of [14].

The remainder of this extended abstract is organized as follows. In Sect. 2 we explain our notation and state several results about regular graphs. Sect. 3 is devoted to the exposition of our main lemma, Lemma 12, the proof of which will appear elsewhere [2]. Roughly speaking, this lemma asserts that for $\varepsilon^{\prime} \gg \varepsilon$, certain subgraphs of $G \in \mathcal{G}\left(K_{\ell}, n, m, \varepsilon\right)$ are $\left(\varepsilon^{\prime}\right)$-regular and have further "typical" properties with very high probability. Suppose the members of a family of bad graphs $\mathcal{B}\left(K_{\ell}, x, y\right) \subseteq \mathcal{G}\left(K_{\ell}, x, y\right)$ are very rare, that is, if we choose a graph $H \in \mathcal{G}\left(K_{\ell}, x, y, \varepsilon\right)$ uniformly at random, then the probability of $H \in \mathcal{B}\left(K_{\ell}, x, y\right)$ is exponentially small for $x$ sufficiently large, $y \geq y_{0}(x)$, and $\varepsilon$ sufficiently small. Now consider a graph $G \in \mathcal{G}\left(K_{\ell}, n, m, \varepsilon\right)$ with $n \gg x$ vertices, and choose subsets $X_{i} \subseteq V_{i}, 1 \leq i \leq \ell$, of size $x$ randomly. The main result of [1] essentially states that for all $\varepsilon^{\prime}>0$, there exists $\varepsilon>0$ such that with high probability the induced graph $G\left[X_{1}, \ldots, X_{\ell}\right]$ contains a large $\left(\varepsilon^{\prime}\right)$-regular subgraph $G^{\prime}$, i.e., $G^{\prime} \in \mathcal{G}\left(K_{\ell}, x^{\prime}, y, \varepsilon^{\prime}\right)$ with $x^{\prime} \sim x$ and $y \sim x^{2} m / n^{2}$. The lemma asserts that $G^{\prime}$ satisfies even more, namely that $G^{\prime} \in \mathcal{G}\left(K_{\ell}, x^{\prime}, y, \varepsilon^{\prime}\right) \backslash \mathcal{B}\left(K_{\ell}, x^{\prime}, y\right)$ provided $x^{2} m / n^{2} \geq y_{0}(x)$. Since the size of the neighborhood into one vertex class of a typical vertex is about $m / n$, we can apply the lemma for $x \approx m / n$ and $\ell-1$ and deduce that most vertices in one class have nicely structured neighborhoods within the other $\ell-1$ classes. This allows the application of the induction hypothesis in the proof of our main result Theorem 6, which is given in Sect. 4.

\section{Notation and Preliminaries}

All graphs are labeled, undirected, and simple, i.e., contain no multiple edges. We denote the vertex set of a graph $G$ by $V(G)$ and its edge set by $E(G)$. For any vertex $v \in V(G)$, we denote its neighborhood by $\Gamma(v)$. For any vertex set $X \subseteq V(G), G[X]$ denotes the graph induced by $X$.

The logarithm is always the natural logarithm. All variables denoted by small greek letters have a tiny value, say, less than $10^{-10}$. We will omit floors and ceilings in order to round expressions like $n / k$ to an integral value. As we assume that $k$ is fixed and $n$ tends to infinity, this does not make any essential difference, but greatly simplifies notation. We write $a \sim_{\varepsilon} b$ if $(1-\varepsilon) b \leq a \leq(1+\varepsilon) b$.

As in Definitions 2 and 4, we use capital, curly letters to denote families of graphs. To simplify notation slightly we shall write $\mathcal{G}(\ell, n, m)$ for $\mathcal{G}\left(K_{\ell}, n, m\right)$ and $\mathcal{G}(\ell, n, m, \varepsilon)$ for $\mathcal{G}\left(K_{\ell}, n, m, \varepsilon\right)$ respectively. We define the family $\widetilde{\mathcal{G}}(\ell, n, m, \varepsilon)$, which is a superset of $\mathcal{G}(\ell, n, m, \varepsilon)$, as follows. $\widetilde{\mathcal{G}}(\ell, n, m, \varepsilon)$ is the family of $\ell$-partite graphs on $\ell$ pairwise disjoint vertex sets $V_{1}, \ldots, V_{\ell}$ of size $n$ such that for all $1 \leq i<j \leq \ell$ the bipartite graph between $V_{i}$ and $V_{j}$

(i) has $m_{i, j} \sim_{\varepsilon} m$ edges and

(ii) is $(\varepsilon)$-regular.

In contrast to $\mathcal{G}(\ell, n, m, \varepsilon)$ the graphs in $\widetilde{\mathcal{G}}(\ell, n, m, \varepsilon)$ do not have a fixed number of edges, but the number of edges may vary by some $\varepsilon$-fraction in every regular pair of vertex classes.

The following theorem from [1] states that most small sets of an $(\varepsilon)$-regular graph also contain a large regular subgraph.

Theorem 9 ([1]) For $0<\beta, \varepsilon^{\prime}<1$, there exist $\varepsilon_{0}=\varepsilon_{0}\left(\beta, \varepsilon^{\prime}\right)>0$ and $C=C\left(\varepsilon^{\prime}\right)$ such that for any $0<\varepsilon \leq \varepsilon_{0}$, every $(\varepsilon)$-regular graph $G=\left(V_{1} \cup V_{2}, E\right)$ with density $d$ satisfies that the number of sets 
$Q \subset V_{1}$ of size $q=|Q| \geq C d^{-1}$ that contain a set $\widetilde{Q}$ of size at least $\left(1-\varepsilon^{\prime}\right)|Q|$ inducing an $\left(\varepsilon^{\prime}\right)$-regular graph of density $d^{\prime} \sim_{\varepsilon} d$ with $V_{2}$, is at least $\left(1-\beta^{q}\right)\left(\begin{array}{c}\left|V_{1}\right| \\ q\end{array}\right)$.

Theorem 9 can be applied to obtain the following consequence [5].

Theorem 10 ([5]) Let $\ell \geq 3$ be any integer. For all $\alpha>0, \beta>0$ and $\varepsilon^{\prime}>0$, there exist constants $\varepsilon_{0}=\varepsilon_{0}(\alpha, \beta, \varepsilon)>0$ and $C=C\left(\varepsilon^{\prime}\right)>0$ such that for all $0<\varepsilon \leq \varepsilon_{0}$, $n$ sufficiently large, and $m \geq C n^{3 / 2}$ all but at most $\beta^{m}\left(\begin{array}{c}n^{2} \\ m\end{array}\right)$ graphs in $\mathcal{G}(\ell, n, m, \varepsilon)$ satisfy that all but $\alpha$ n vertices $v$ in $V_{1}$ have neighborhoods that contain a graph in $\mathcal{G}\left(\ell-1, x, y, \varepsilon^{\prime}\right)$ with $x=\left(1-\varepsilon^{\prime}\right) m / n$ and $y=\left(1-\varepsilon^{\prime}\right) x^{2} m / n^{2}$.

\section{Typical Tuples of Sublinear Subsets}

Before we state the main result of this section, let us discuss some consequences of the inheritance property of $\varepsilon$-regularity stated in Theorem 9. It was proved in [5] that given $\varepsilon^{\prime}>0$ and $\beta>0$ we can find $\varepsilon>0$ and $C>0$ such that all graphs in $\mathcal{G}(\ell, n, m, \varepsilon)$ satisfy for all $x \geq C n^{2} / m$ the following property: if we choose sets $X_{i} \subseteq V_{i}$ of size $\left(1+\varepsilon^{\prime}\right) x$ randomly, then with probability $1-\beta^{x}$ these sets contain a graph from $\widetilde{\mathcal{G}}\left(\ell, x, x^{2} m / n^{2}, \varepsilon^{\prime}\right)$.

In this section we want to generalize this result as follows. Instead of just requiring that the graph induced by the tuple $\left(X_{1}, \ldots, X_{\ell}\right)$ contains a regular subgraph that has approximately the expected number of edges, we want to deduce that it also has further "typical" properties. Here we call a property "typical" if for suitable $n$ 's and $m$ 's, all but a $\beta^{m}$-fraction of the graphs in $\mathcal{G}(\ell, n, m, \varepsilon)$ satisfy it. We formalize this as follows.

Definition 11 We say that a family $\mathcal{B}(\ell, n, m) \subseteq \mathcal{G}(\ell, n, m)$ is small with respect to a function $m_{0}(n)$ if for all $\beta>0$, there exist constants $n_{\beta} \in \mathbb{N}, C_{\beta}>0$, and $\varepsilon_{\beta}>0$ such that

$$
\left|\mathcal{B}(\ell, n, m) \cap \mathcal{G}\left(\ell, n, m, \varepsilon_{\beta}\right)\right| \leq \beta^{m} \cdot\left(\begin{array}{c}
n^{2} \\
m
\end{array}\right)^{\left(\begin{array}{c}
\ell \\
2
\end{array}\right)} \quad \forall n \geq n_{\beta}, m \geq C_{\beta} m_{0}(n) .
$$

Thus our main result Theorem 6 states that for $\ell>3$ and $\delta>0$, the family $\mathcal{F}(\ell, n, m, \delta)$ is small with respect to $n^{2-1 /(\ell-1)}$. The key for proving this theorem will be our next lemma.

Lemma 12 ([2]) Let $y_{0}(x) \geq x$ be a monotone increasing function and let $\mathcal{B}(\ell-1, x, y)$ be small with respect to $y_{0}$. For all $\beta, \varepsilon^{\prime}>0$, there exist constants $\varepsilon_{0}>0$ and $C>0$ such that for all $\varepsilon$, $x$, and $y$ satisfying

$$
0<\varepsilon \leq \varepsilon_{0}, \quad x=\left(1-\varepsilon^{\prime}\right) \frac{m}{n}, \quad m \gg n^{3 / 2} \sqrt{\log n}, \quad C y_{0}(x) \leq \frac{m^{3}}{n^{4}}, \quad \varepsilon^{\prime} \frac{m^{3}}{n^{4}} \leq y \leq\left(1-\varepsilon^{\prime}\right)^{3} \frac{m^{3}}{n^{4}},
$$

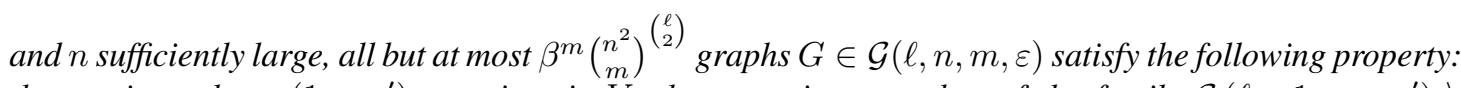
there exist at least $\left(1-\varepsilon^{\prime}\right) n$ vertices in $V_{1}$ that contain a member of the family $\mathcal{G}\left(\ell-1, x, y, \varepsilon^{\prime}\right) \backslash$ $\mathcal{B}(\ell-1, x, y)$ in their neighborhood. 


\section{Proof of Theorem 6}

Before we come to the proof of Theorem 6 , we shall proof the base case $\ell=3$ of the induction.

Lemma 13 For all $\delta>0$, the family $\mathcal{F}(3, n, m, \delta)$ is small with respect to $m_{3}=n^{3 / 2}$.

Proof: Let $\delta^{\prime}>0$ satisfy $(1-\delta) \leq\left(1-\delta^{\prime}\right)^{4}$. Apply Theorem 10 with parameters $\ell \leftarrow 3, \alpha \leftarrow \delta^{\prime}, \delta \leftarrow \delta^{\prime}$, and $\beta$. Hence, we obtain constants $\varepsilon_{0}>0$ and $C>0$ such that for all $0<\varepsilon \leq \varepsilon_{0}, m \geq C n^{3 / 2}$, and $n$ sufficiently large, all but at most $\beta^{m}\left(\begin{array}{c}n^{2} \\ m\end{array}\right)^{3}$ graphs $G \in \mathcal{G}(3, n, m, \varepsilon)$ satisfy that at least $\left(1-\delta^{\prime}\right) n$ vertices $v$ in $V_{1}$ have neighborhoods containing a bipartite graph with at least $\left(1-\delta^{\prime}\right)^{3} m^{3} / n^{4}$ edges. Since each edge forms a triangle with $v$, the number of triangles in $G$ is at least $\left(1-\delta^{\prime}\right)^{4} n m^{3} / n^{4} \geq(1-\delta) m^{3} / n^{3}$.

Proof of Theorem 6: We prove the theorem by induction on $\ell$. The base case is settled by Lemma 13. So assume that the Theorem holds for some $\ell-1 \geq 3$, i.e., for all $\delta^{\prime}>0$, the family $\mathcal{F}\left(\ell-1, x, y, \delta^{\prime}\right)$ is small with respect to $y_{\ell-1}(x)=x^{2-1 /(\ell-2)}$. Choose $\delta^{\prime}>0$ such that $\left(1-\delta^{\prime}\right)^{\left(\begin{array}{l}\ell \\ 2\end{array}\right)+2} \geq 1-\delta$. Apply Lemma 12 with the family $\mathcal{F}\left(\ell-1, x, y, \delta^{\prime}\right)$ and the parameters $\beta$ and $\varepsilon^{\prime} \leftarrow \delta^{\prime}$ in order to obtain constants $\varepsilon_{0}>0$ and $C_{\ell-1}>0$. Let $C=C_{\ell-1}$. Observe that $m \geq C n^{2-1 /(\ell-1)} \geq C n^{5 / 3} \gg n^{3 / 2} \log n$ since $\ell \geq 4$, and that

$$
C_{\ell-1} y_{\ell-1}(x)=C_{\ell-1}\left[\left(1-\delta^{\prime}\right) \frac{m}{n}\right]^{\frac{2 \ell-5}{\ell-2}} \leq C_{\ell-1} m^{-\frac{\ell-1}{l-2}} n^{\frac{2 \ell-3}{\ell-2}} \frac{m^{3}}{n^{4}} \leq C \frac{m^{3}}{n^{4}}
$$

since $m \geq C n^{2-1 /(\ell-1)}$. Thus, for $y=\left(1-\delta^{\prime}\right)^{3} m^{3} / n^{4}$ and $n$ sufficiently large, all but at most $\beta^{m}\left(\begin{array}{l}n^{2} \\ m\end{array}\right)$ ( $\left.\begin{array}{c}\ell \\ 2\end{array}\right)$ graphs $G \in \mathcal{G}(\ell, n, m, \varepsilon)$ satisfy the following property: there are at least $\left(1-\delta^{\prime}\right) n$ vertices $v \in V_{1}$ that contain a member of the family $\mathcal{G}\left(\ell-1, x, y, \delta^{\prime}\right) \backslash \mathcal{F}\left(\ell-1, x, y, \delta^{\prime}\right)$ in their neighborhood. But this means that each such vertex $v$ has at least $\left(1-\delta^{\prime}\right) x^{\ell-1}\left(y / x^{2}\right)^{\left(\begin{array}{c}\ell-1 \\ 2\end{array}\right)}$ cliques of size $\ell-1$ in its neighborhood. Hence, the number of complete graphs on $\ell$ vertices in $G$ is at least

$$
\begin{aligned}
\left(1-\delta^{\prime}\right) n\left(1-\delta^{\prime}\right) x^{\ell-1}\left(\frac{y}{x^{2}}\right)^{\left(\begin{array}{c}
\ell-1 \\
2
\end{array}\right)} & =\left(1-\delta^{\prime}\right)^{2} n\left[\left(1-\delta^{\prime}\right) \frac{m}{n}\right]^{\ell-1}\left[\left(1-\delta^{\prime}\right) \frac{m}{n^{2}}\right]^{\left(\begin{array}{c}
\ell-1 \\
2
\end{array}\right)} \\
& =\left(1-\delta^{\prime}\right)^{\left(\begin{array}{c}
\ell-1 \\
2
\end{array}\right)+\ell+1} n^{\ell}\left(\frac{m}{n^{2}}\right)^{\left(\begin{array}{c}
\ell-1 \\
2
\end{array}\right)+\ell-1} \geq(1-\delta) n^{\ell}\left(\frac{m}{n^{2}}\right)^{\left(\begin{array}{c}
\ell \\
2
\end{array}\right)}
\end{aligned}
$$

As mentioned in the introduction, the threshold $m \geq n^{2-1 /(\ell-1)}$ can be improved by lifting the base case of the induction. If we used $\ell=4$, the induction would work for $m \geq n^{2-1 /(\ell-3 / 2)}$. The case $\ell=5$ yields $m \geq n^{2-1 /(\ell-2)}$ and so forth. Rough calculations reveal that in order to prove the conjectured threshold $m \geq n^{2-2 /(\ell+1)}$ inductively, one has to use the induction assumption $\ell-2$ instead of $\ell-1$.

\section{References}

[1] S. Gerke, Y. Kohayakawa, V. Rödl, and A. Steger. Small subsets inherit sparse $\varepsilon$-regularity. submitted, 2004.

[2] S. Gerke, M. Marciniszyn, and A. Steger. A probabilistic counting lemma for complete graphs. submitted, 2005. 
[3] S. Gerke, H.J. Prömel, T. Schickinger, A. Steger, and A. Taraz. $K_{4}$-free subgraphs of random graphs revisited. submitted, 2002.

[4] S. Gerke, T. Schickinger, and A. Steger. $K_{5}$-free subgraphs of random graphs. Random Structures \& Algorithms, 24(2):194-232, 2004.

[5] S. Gerke and A. Steger. The sparse regularity lemma and its applications. In 20th British Combinatorial Conference (Durham, UK, 2005), 2005.

[6] S. Janson, T. Łuczak, and A. Rucinski. Random graphs. Wiley-Interscience Series in Discrete Mathematics and Optimization. Wiley-Interscience, New York, 2000.

[7] Y. Kohayakawa. Szemerédi's regularity lemma for sparse graphs. In Foundations of computational mathematics (Rio de Janeiro, 1997), pages 216-230. Springer, Berlin, 1997.

[8] Y. Kohayakawa, T. Łuczak, and V. Rödl. On $K^{4}$-free subgraphs of random graphs. Combinatorica, 17(2):173-213, 1997.

[9] Y. Kohayakawa and V. Rödl. Regular pairs in sparse random graphs. I. Random Structures \& Algorithms, 22(4):359-434, 2003.

[10] Y. Kohayakawa, V. Rödl, and M. Schacht. The Turán theorem for random graphs. Combin. Probab. Comput., 13(1):61-91, 2004.

[11] J: Komlós, A. Shokoufandeh, M. Simonovits, and E. Szemerédi. The regularity lemma and its applications in graph theory. In Theoretical aspects of computer science (Tehran, 2000), volume 2292 of Lecture Notes in Comput. Sci., pages 84-112. Springer, Berlin, 2002.

[12] J. Komlós and M. Simonovits. Szemerédi's regularity lemma and its applications in graph theory. In Combinatorics, Paul Erdôs is eighty, Vol. 2 (Keszthely, 1993), volume 2 of Bolyai Soc. Math. Stud., pages 295-352. János Bolyai Math. Soc., Budapest, 1996.

[13] T. Łuczak. On triangle-free random graphs. Random Structures \& Algorithms, 16(3):260-276, 2000.

[14] T. Szabó and V. H. Vu. Turán's theorem in sparse random graphs. Random Structures \& Algorithms, 23(3):225-234, 2003.

[15] E. Szemerédi. Regular partitions of graphs. In Problèmes combinatoires et théorie des graphes (Colloq. Internat. CNRS, Univ. Orsay, Orsay, 1976), volume 260 of Colloq. Internat. CNRS, pages 399-401. CNRS, Paris, 1978. 
